\title{
Zur Theorie der Zustandsgleichungen.
}

\author{
Von
}

M. v. Smoluchowski.

Separat-Abdruck aus den

Annąlen der Physik.

Vierte Folge. Band 48.

1915.

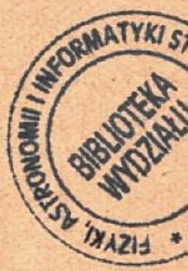

\section{Loipzig,}

Johann Ambrosius Barth. 
RRYK, OTTO. Entwicklungsgeschichte der reinen und angewandten Naturwissen-

schaft im XIX. Jahrhundert. I. Band: Die Naturphilosophie und ihre Überwindung durch die erfahrungsgemäße Denkweise (1800-1850). XL, 655 Seiten. 1909.

M. 15.-, geb. M. 16.-

Literarisches Zentralblatt für Deutschland: Hier liegt eins jener monumentalen Werke vor, die man in einer kurzen Anzeige unmöglich würdigen kann. Es ist die Absicht des Verfassers, den einzigartigen Entwicklungsgang der gegenwärigen Naturforschung in einheitlichem geschichtliclen Bilde darzustellen. Der gewaltige Stott. ist in zwei größere Bände aufgeteilt, die dazu bewimmt sind, die zwoi aufeinanderfolgenden Kichtungen in der naturwissenschaftlichen Tätigkeit des 19. Jahrhunderts gegeneinander aluzugrenzen. Der hier vorliegeude erste Band relcht. bis zur Entdeckung der Krafteinheit durcb Robert Mayer und Helmholtz und zeigt die größte Höhe, zu der sich die Naturforschung wäbrend ibres Kampfes gegen die lang nachwirkenden Einfüsse des rein begrifflichen, erfahrungsarmen Denkens aufschwingt. Nach Erscheinen des zweiten Bandes soll suf das hochbedeutsame Werk, nit dem sich nur etwa Whewells und Apelts Schrifton vergleichen lassen, ausfuhrli, h eingegangen werden.

JEEMAN, P., Magnetooptische Untersuchungen mit besonderer Berucksichtigung

der magnetischen Zerlegung der Spektrallinien. Deutsch von Dr. Ir ax Iklé. XI, 242 Seiten mit 74 Abbildungen im T'ext und 8 Lichtdrucktafeln. 1914.

M. 8. - , geb. M. 9.-

Zeitschrift für den physikalisehen und chemischen Unterricht: Das Werk enthalt im weitesten Sinne das Gebiet, in welchem Zeewan seine epochemachenden Forschungen angestellt hat. Da diese erst durch das hohe Auflösungevermögen der modernen Spektroskope ermöglicht wurden, so ist es sehr dankenswert, daß die entsprechenden Apparate von Rowland, Michelson, Fabry und Perot eingthend beschrieben werden. AuBer der ausführlichen Darstellung des Zeeman'scben Phänomens in allen seinen Formen gibt der Verfasser auch einen Einblick in die Gebiete, die eng mit der magnetischen Auflósuug der Absorptionslinien verknüpft siud: die magnetische Drehung der Polarisationsebeno und dis magnetische Doppeldrehung. In oinem besonderen Kapitel wird angezelgt, wie die magnetische Auflösung der Spektsallinien auch ein Licht wirft auf die Konstitution der Afome. Sehr dankenswert ist das Jiteraturverzeichnis, das nicht nur de Abhandlungen Zeemans, sondern auch alle anderen in den verschiedenen Landern von 1856 bis 1913 in diesem Gebiet erschienenen A rbeiten enthålt. Das Werk Ist für den Fachmann ron böchstem Interesse; wegen der leichtrerständlichen Darstellung ist. es auch zur Einführung in dleses immer wichtiger werdende Gebiet der Physik durchaus geeignet.

ahrbuch der drahtlosen Telegraphie und Telephonie sowie des Gesamtgebietes der elektromagnetischen Schwingungen. Unter Mitarbeit von vielen Gelehrten und unter bes. Mitwirkung von Prof. Dr. J. Zenneck, herausgegeben von Dr. G. Eichhorn. Preis des Bandes von 6 Heften. M. 20.-

Das Jahrbuch bringt theoretische und praktische Arbeiten ủber die Anwendung der drahtlosen Telegraphie und zwar nicht nur Originale, sondern auch kritische Besprechungen in- und ausländischer Patente, Sammelberichte und Beschreibungen von technischen Ausnihrungen und Anlagen, alles mit absoluter Objektivitat. Band $\mathrm{X}$ ist im Erscheinen begriffen.

NAIRZ, OTT0, Einfïhrung in die Elektrotechnik. Unter Zugrundelegung der Vorlesungen Prof. Slabys. VIII, 415 Seiten mit 351 Abbildungen im Text. 1913.

M. 10.-, geb. M. 11.-

Das rorliegende, elementar gehaltene Lehrbuch der Elektrotechntk ist aus den Vorlesungen entsianden, wolche ler Verfasser in ministeriellen Auftrag als Vertreter seiues beurlaubten Chefs, Herrn Geheimen Regierungsrat Professor Dr. Dr.-Ing. A dolf Slaby, während $3^{1}$, Semester an der Königl. Technischen Hochschule Berlin halten durfıe. Die Unterlagen hierzu rühren zum gröBten Telle von Slab selbut her, teils aus Niederschriften, die der Verfasser witurend Beiner Vortrige machte, teils nach den Konzepten derselben, welche der Dahingegangene ihm zum Zwecke der Veröffentlichung zur Verfügung stellte. An der Korrekfur des Manuskriptes hat er sich sogar selbst beteiligt.

Der Verfasser hat sich bei der Niederschrift eng an die ungemein pädagogische Lehrkunst $S$ lab y gehalten und das Werk dürte dahor nicht nur den vlelen Schalern Slabys hochwillkoumen sein, sondern sich auch sonst als eine elementare Einführung in die lifictrotechnik bewihren.

ODGE, SIR OLIVER. Radioaklivität und Kontinuität. Zwei Vorträge: I. Die Entdeckuug der Radioaktivitat und deren Einfluß auf die Entwicklung der Physikalischen Wissenschaft. Becquerel Gedächtnisrede, gehalten am 17. Oktober 1912 vor der Chemical Society. - II. Kontinuität. Eröffnungsrede, gehalten auf der Versammlung der British Association zu Birmingham 1913. IV, 217 Seiten. 1914. M. 5-, geb. M. 6.-

Diese beiden Vorträge des gloßcu englischen Physikers bebandeln Fragen, die weit aber den Kreis der Fachgonossen hinaus das lebhafteste Interesse fôr sich in Anspruch nchımen müssen. Im ersten Vortrag legt der Verfasser dar, wie die Entdeckung der Radioaktiritst auf unser naturwissenschaftliches Denken bestimmend wirkt. Im zweiten Vortrag legt der Verfasser sein physikalisches Glaubensbekenntnis ab. Sicberlich werden die interessanten Ausfuhrungen des Verfassers und seine vielseitigen Darlegungen Anhänger und Gegner in gleichem Maße fenseln und anregen. 


\section{Zur Theorie der Zustandsgleichungen; von M. v. Smoluchowski.}

Unter obigem Titel hat M. B. Wagner in diesen Annalen eine Arbeit ${ }^{1}$ ) veröffentlicht, deren Hauptzweck der Nachweis ist, daß van der Waals, Boltzmann, Kuenen und alle anderen Autoren, welche die Wirkung der molekularen Anziehungskräfte durch Einführung eines inneren Druckes $a / v^{2}$ ersetzten, in einem Irrtum befangen waren, indem sich bei korrekter Berechnung unter den van der Waalsschen Voraussetzungen vielmehr eine Zustandsgleichung von der Gestalt:

$$
p v=r T e^{-\frac{f(v)}{r T}}\left[1+\frac{b}{v} e^{-\frac{f(v)}{r T}}+\ldots\right]
$$

ergebe. Insbesondere richtet sich die Spitze jener Ausführungen gegen Boltzmann, welcher bei seinen Bestrebungen, die Ableitung der vander Waalsschen Gleichung in möglichst präziser Form zu geben, gewisse Fehler begangen haben soll.

Nun glaube ich, daß diese schweren Vorwürf auf Mißverständnisse zurückzufüren sind, und daß die herkömmliche Auffassung vollkommen zu Recht besteht. Gewisse, bei der Bgründung jener Gleichung zutage tretende Schwierigkeiten verdienen aber vielleicht tatsächlich eine eingehendere Analyse, als meistens üblich ist, und deshalb scheint es mir nützlich, die Sachlage behufs Klarlegung der Einwände Wagners in möglichst einfach $r$ Weise kurz auseinanderzusetzen.

Um das Wesentliche hervortreten zu lassen, seien von vornherein Punktmoleküle angenommen, wodurch die Figenvolum-Korrektion wegfällt. Als Druck des Gases ist nun nach Wagner die Bewegungsgröße anzusehen, welche die Molekularstöße auf eine unęndlich dünne Wand übertragen, welche das Gas gegen den leeren Raum abgrenzt; somit ist in bekannter Weise der Druck:

$$
p=\frac{n_{w} m c^{2}}{3}
$$

1) M. B. Wagner, Ann. d. Phys. 45. p. 1169.1914. 
wobei $n_{w}$ die Molekülzahl bedeutet, welche in der unmittelbar der Wand anliegenden Schicht pro Volumeinheit enthalten ist. Letztere Molekülzahl ist aber nicht mit der im Inneren des Gasraumes (Wagners "Stoffkern“) pro Volumeinheit entfallenden Zahl $n_{0}$ identisch, da die Gasdichte in den Grenzschichten mit Annäherung an die Wand abnimmt. Und zwar

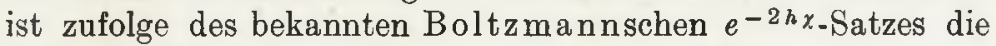
Molekülzahl in der Entfernung $x$ von der Wand:

$$
n(x)=n_{0} e^{-2 h \int_{x}^{\infty} f(\xi) d \xi},
$$

wo $F(\xi)$ die nach dem Gasinneren gcrichtete Resultante der Kohäsionskräfte bedeutet, welche auf ein im Abstande $\xi$ von der Wand befindliches Molekül seitens der übrigen Gasmoleküle ausgeübt werden. Führt man für die zum Transport eines Moleküls vom Inneren an die Wand erforderliche Arbeit die Abkürzung ein:

$$
\int_{0}^{\infty} F(\xi) d \xi=A,
$$

so geht also (2) mit Rücksicht auf (3) und die Relationen

über in

$$
c^{2}=3 r T, \quad 2 h=\frac{N}{R T}
$$

$$
p=\frac{r T}{v} e^{-\frac{N}{R} T^{A}} .
$$

Soweit ist also Wagners Ableitung ${ }^{1}$ ) ganz richtig, aber unbegründet ist dessen Ansicht, daß $A$ blo $B$ eine Funktion des spezifischen Volums $v$ sei, da ja die Kräfte $F(\xi)$ im allgemeinen von der Molekülverteilung in den Wandschichten, also der Veränderlichkeit der $n(\xi)$ abhängen und letztere durch die in $h$ enthaltene Temperatur mit bestimmt wird.

Betrachten wir diese Verteilung etwas näher. Durch Differentiation von (3) erhält man

$$
\frac{1}{n} \frac{d n}{d x}=2 h F(x)
$$

was mit der acrostatischen Grundgleichung identisch ist. Nun läßt sich $F(x)$ in die Anziehungskräfte zerlegen, welche das

1) Unsere Bezeichnungsweise weicht etwas von jener Wagners ab und schließt sich mehr an Boltzmann an. 
in $x$ befindliche Molekül seitens der in verschiedenen Entfernungen $\xi$ von der Wand befindlichen Gasschichten (von der Dicke $d \xi$ ) erfährt. Dieselben lassen sich in der Form $n(\xi) \Psi(\xi-x) d \xi$ ausdrücken, wo die Funktion $\Psi$ bloß von der Gestalt des Kraftgesetzes abhängt; dieselbe ist natürlich eine unpaare Funktion und muß im Sinne der Boltzmannschen Voraussetzungen als in einem relativ großen Wirkungsbereich $\left.{ }^{1}\right) \quad|\xi-x|<\varrho_{2}$ wenig veränderlich, aber für makroskopische Entfernungen als verschwindend klein von hoher Größenordnung angenommen werden. Die Verteilung der Moleküle ist also durch die Differential-Integralgleichung bestimmt:

$$
-\frac{1}{n} \frac{d n}{d x}=2 h \int_{0}^{\infty} n(\xi) \Psi(\xi-x) d \xi .
$$

Ohne in eine genauere Analyse derselben einzugehen, kann man ein für unseren Zweck genügendes Resultat ableiten, indem man mit $n$ multipliziert und nach $d x$ zwischen den Grenzen Null und $x$ integriert, wodurch sich ergibt:

$$
n(x)-n_{w}=2 h \int_{0}^{x} \int_{0}^{\infty} n(x) n(\xi) \Psi(\xi-x) d x d \xi .
$$

Nun verschwindet aber offenbar die Resultierende der Kohäsionskräfte aller zwischen 0 und $x$ gelegenen Moleküle infolge der Gleichheit von Wirkung und Gegenwirkung:

$$
\int_{0}^{x} \int_{0}^{x} n(x) n(\xi) \Psi(\xi-x) d x d \xi=0
$$

und es verbleibt:

$$
u(x)-n_{w}=2 h \int_{u}^{x} \int_{x}^{\infty} n(x) n(\xi) \psi(\xi-x) d x d \xi .
$$

Verlegt man jetzt den Punkt $x$ ins Innere des Gases, wo die gleichförmige Dichte $n_{0}$ herrscht, so wird

$$
n_{u}=n_{0}-2 h n_{0}^{2} \varepsilon,
$$

1) Boltzmann verlangt bekanntlich, da $\beta$ der Bereich $\varrho_{2}$ gro $\beta$ sei im Verhältnis zum mittleren Molekularabstand. Eine noch etwas weiter gehende Bedingung erhält man, wenn die zufälligen Dichteschwankungen des Gases berücksichtigt werden. Vgl. M. v. Smoluchowski, BoltzmannFestschrift, p. 626. 1904. In Wirklichkeit dürften diese Voraussetzungen wohl nicht zutreffen. 
indem das Doppelintegral

$$
\int_{0}^{x} \int_{x}^{\infty} \Psi(\xi-x) d x d \xi=\alpha
$$

gesetzt wird, was in (2) eingesetzt, unter Benutzung der Abkürzung $a / m^{2}=a$ die übliche Formel ergibt:

$$
p+\frac{a}{b^{2}}=\frac{r T}{v} .
$$

Dieselbe bleibt also im Gegensatz zu Wagners Behauptung auch in der strengen Theorie gültig, welche der Veränderlichkeit der Dichte in den Grenzschichten Rechnung trägt. Da dieselbe mit (4) nicht in Widerspruch stehen kann, läßt sich nun umgekehrt für die Arbeit $A$ die Beziehung ableiten:

$$
A=-\frac{R T}{N} \log \left(1-\frac{a}{v r T}\right)
$$

Die Richtigkeit der Gleichung (10) läßt sich übrigens in ganz einfacher Weise auch folgendermaßen beweisen. Im Gleichgewichtszustand muß die seitens eines Gases von bestimmter Dichte auf den Flächeninhalt der umgebenden Wände ausgeübte Kraft aus thermodynamischen Gründen unabhängig sein von der Art der Wand, wie auch Wagner am Schlusse seiner Arbeit ausführt. Denken wir uns also eine Wand von erheblicher Dicke und von genau derselben Dichte und Molekularkraft, wie jene des Gases, so daß die Wand in bezug auf die von ihr ausgeübten Anziehungskräfte durch jenes Gas ersetzbar sei.

Dann bleibt die Gasdichte in den Wandschichten gleich jener im Inneren; also steht die Wand unter der Wirkung der Stoßkräfte: $\frac{n_{0} m c^{2}}{3}$ und der entgegengesetzten, vom Gase ausgehenden Kohäsionskräfte, deren Resultante offenbar dem Quadrat der Dichte proportional ist, sonst aber nur von der Form des Kraftgesetzes abhängt [entsprechend dem Doppelintegral in (9)]. Dies ergibt sofort die Formel (10).

Boltzmann setzt sich allerdings über die Schwierigkeiten, welche die Behandlung der Grenzschichten mit sich bringt, kurz hinweg, doch scheint mir seine auf der Benutzung des Virials beruhende Ableitung ${ }^{1}$ ) im Gegensatz zu Wagners An-

1) L. Boltzmann, Gastheorie 2. p. 152. 1898.

Annalen der Physik. IV. Folge. 48. 
1102 M. v. Smoluchowski. Zur Theorie der Zustandsgleichungen.

sicht ebenfalls ganz einwandfrei zu sein. Wird nämlich das Virial in der Form $\Sigma(x X+y Y+z Z)$ angewendet, so gibt allerdings das Innere des Gases (der Stoffkern) keinen Beitrag zu diesem Ausdruck, und es bleiben als ausschlaggebend gerade nur die durch veränderliche Dichte charakterisierten Wandschichten übrig.

Verwendet man dasselbe dageg $\mathrm{n}$ in der Form $\Sigma r f(r)$, so summieren sich die betreffenden Beiträge der Moleküle des Stoffkernes, und die Wandschichten kommen als verschwindend dünn gar nicht in Betracht - das ist eben der ganze Vorteil dieser von Boltzmann angenommenen Berechnungsmethode. Es sind nämlich bei der üblichen Methode der Summierung jene zwei Formen für ein von inneren Kräften beeinflußtes endliches System zwar im ganzen äquivalent, nicht aber für willkürliche abgegrenzte T'eile desselben. Wagner ist somit in einem Irrtum befangen, wenn er meint, daß $\Sigma r f(r)$ für das Gasinnere verschwinde, und damit entfällt auch $\mathrm{s}$ in gegen Sutherlands und Bakkers Ableitung vorgebrachter Einwand.

Das Gesagte dürfte zur Klarstellung der Sache genügen. Die Physiker, welche seit van der Waals' Abhandlung den von diesem Forscher eingeschlagenen Gedankengang sich angeeignet haben, sind dabei meistens mehr einem intuitiven physikalischen Gefühl, als ganz exakter mathematischer U̇berlegung gefolgt. Wir können mit Beruhigung konstatieren, da $B$ sie von jener Intuition nicht irregeleitet worden sind.

Krakau, 15. November 1915.

(Eingegangen 25. November 1915.) 

1 UERBACH. F, Geschichtstafein der Physik. V, 150 Seiten. 1910.
M. 4.-, geb. M. 5.

Zeitschrift für die osterreichischen Gymnasien: Das Buch, das mit vieler'Sorgfalt und guten Geschicke verfalit jst, wird sich recht brauchuar erweisen, z. B. für Vorlesungen, zur Vorbereitung auf Profungen, zur Entschelduug historischer Fragen; dann wird auch das Buch wie der Verfasser mit vollem Rechte bemerkt - für den, der in und $z$ wischon den Zeilen zu lesen versteht, eino anregende und fesselnde Lektüre bilden. Wir wünschen dem sehr belangrefchen Ruche oine weite Verbreitung.

NAIRZ, 0., Die elektrische Arbeitsübertragung. VII, 260 Seiten mit 144 Abb1909.

Vorliegendes Buch wendet sich in erster Linie an den Laien und Lernenden, ohne mehr als die einfachsten Kenntnisse voraliszusetzen. Obne viel Mưhe sucht es ihm das Notwendige grūndlich klar zu machen und ihn in die Lage zu bringen, Vorteile und Nachteile der einzelnen Systeme gegenüber einem gegebenen Fall abwägen zu können.

STWaLD, W., Die Energie. 2. Auff. 167 Seiten. 1912.

Geb. M. 4.40.

Münchner Neueste Nachrichten: Erst kürzilich ist an dieser Stelle auf ein ähnliches Werk Ostwalds hingewiesen, in welchem er das Worden einer Wigsenscbaft schildert. In diesem neuesten Buch nun schildert er in gleich hervorragender Weise das Werden der Energie unl des Lebens, das Walten der Energie in allen Lebens- und Umformungsersebcinungen; und das mit einer Meisterschaft, in einer Sprache, die bewundernswert ist. Es ist eive Lust, ein solches Buch zu lesen.

H

UGHES, ARTHUR LLEWELYN, Die Lichtelektrizität.

I klé.

192 Seiten mit 40 Figuren. 1915.

Dentsch von Dr. Max sehr geschickte Zusammenfassung der neuesten Forschungen enthâlt, die auf diesem modernsten Gebiete der Physik angestellt worden aind.

$\mathrm{Da}$ seit 5 Jahron keine vollstundige Zusammenfassung uber den behandelten Gegenstand erschienen ist, inzwischen aber erhebliche Fortschritte auf dem Gebiete erzielt worden sind, dürfte die Übersetzung Anklang finden. Der Verfasser bat dabel alle Formen der Ionisation durch Licht, sei es in festen, in flüssigen oder in gasformigen Körpern, in den Kreis seiner Betrachlungen gezogen.

REIS, PAUL, Elemente der Physik, Meteorologie und mathenatischen Geographie. Hilfsbuch für den Unterricht an höheren Lehranstalten. Mit zalılieichen Übungsfragen und -Aufgaben. 7., vollständig umgearbeitete Auflage. Herausgegeb von Ed.Penzold. Mit 435 Textfig. X, 419 S. 1905̃. M. 4.80

HOPPE, FRITZ, Wie stellt man Projekte, Kostenanschläge und Betriebskostenberechnungen fïr elektrische Licht- und Kraftanlagen auf? 6. vollständig umgearbeitete Auflage. X, 580 Seiten mit 290 Abbild. 1914. Geb. M. 8.-

Nach wenigen Jahren macht sich wieder eine neue, die 6. Auflage dieses Buches notwendig, das beste Zeichen, daß ein Bedürfnis nach einem derartigen Ratgeber für d. n A kquisiteur und den projektierenden Ingenieur vorliegt und daß das Buch diesem Beđürfnisse auch gerecht wird. Der erste Teil gibt einen Wogweiser, wie man Projekte und elektrische licht- und Kraftanlagen aufetelit. Der zweite und dritte Teil enthalt Rechnungen von Betriebskosten und Rentabilităten, der fünfte Teil Durchschnittsprelse für die einzelnen Teile elektrischer Anlagen usw., sowie uber die Montagekosten. Tabellen und Sachregister bilden den Schluß.

Die neue Auflage enthält ganz wesentliche Veränderungen und Verrollstandigungen, unnötiger Ballast ist entfernt. Die Zahl der Abbildungen isı wesentlich vermehrt.

RIES, CHR., Das Licht in seinen elektrischen und magnetischen Wirkungen. Versuchsergebnisse, Theorien und Literatur. IV, 258 Seiten mit $62 \mathrm{Abb}$. 1909.

Geb. 5. -

Der Verf. hat sich die Aufgabe gestellt, eine Gesamtdargtellung der elektrischen und magnetischen Wirkungen des Lichtes zu geven; die Arbeiten über die rerschiedenen Arten lichtelektrischer Erscheinungen haben sich besonders in den letzten Jahren derariig gehäuft, daß eine ubersichtliche Zusammenstellung aller wesentlichen Versuchsergebnisse und der gesamten Literatur manchem nicht unerwünscht sein dürfte.

QNYDER, CARL, Das Weltbild der modernen Naturwissenschait nach den Ergebnissen der neuesten Ferschungen. Autorisierte deutsche Übersetzung von Hans Kleinpeter. 2. Auf. XII, 306 Seiten. Mit 16 Porträts. 1907. M. 5.60 , geb. M. 6.60 .

Zeitschrift fur den physik. und chem. Unterricht: Das Buch ist schon als eine zusammenfassende Ubersicht aber die ueuesten Forschungen ron Interesse. Es behandeli in populärer Form und zugleich mit sachllcher Genauigkeit die Lehre von den Strahlungen usw. Noch interessanter, weil bei uns weniger bekannt, sind die Forschungen amerikanischer Physjologen (Loeb, Mattbewg) uber die Einwirkung anorganischer Agentien uf die Lebensvorgånge, so die Befruchtung von Seeigeleiern durch Magnesiumehlerid u. a. m. Die letzten Kapitel des Buches behandeln die Grundlagen der Serumpathologie und die Erfadung der drahtlosen Telegraphie. Alles in ullem ein Buch, das über die Probleme, die die heutige Wissemschaft beschaftigen, die mangigfachste Belehrung bietet. 